\title{
Weak and Strong Solutions for a Strongly Damped Quasilinear Membrane Equation
}

\author{
Jin-soo Hwang \\ Department of Mathematics Education, College of Education, Daegu University, Jillyang, Gyeongsan, Gyeongbuk, Republic of Korea \\ Correspondence should be addressed to Jin-soo Hwang; jshwang@daegu.ac.kr
}

Received 9 February 2017; Accepted 4 April 2017; Published 11 June 2017

Academic Editor: Sining Zheng

Copyright (C) 2017 Jin-soo Hwang. This is an open access article distributed under the Creative Commons Attribution License, which permits unrestricted use, distribution, and reproduction in any medium, provided the original work is properly cited.

\begin{abstract}
We consider a strongly damped quasilinear membrane equation with Dirichlet boundary condition. The goal is to prove the wellposedness of the equation in weak and strong senses. By setting suitable function spaces and making use of the properties of the quasilinear term in the equation, we have proved the fundamental results on existence, uniqueness, and continuous dependence on data including bilinear term of weak and strong solutions.
\end{abstract}

\section{Introduction}

Let $\Omega$ be an open bounded set of $\mathbf{R}^{n}$ with the smooth boundary $\Gamma$. We set $Q=(0, T) \times \Omega, \Sigma=(0, T) \times \Gamma$ for $T>0$. The nonlinear equation of the longitudinal motion of vibrating membrane surrounding $\Omega$ with clamped boundary is described by the following Dirichlet boundary value problem:

$$
\begin{aligned}
\frac{\partial^{2} y}{\partial t^{2}}-\nabla \cdot\left(\frac{\nabla y}{\sqrt{1+|\nabla y|^{2}}}\right)-\mu \Delta \frac{\partial y}{\partial t} & =f \quad \text { in } Q, \\
y & =0 \text { on } \Sigma, \\
y(0, x) & =y_{0}(x), \\
\frac{\partial y}{\partial t(0, x)} & =y_{1}(x)
\end{aligned}
$$

in $\Omega$,

where $y$ is the height of a membrane, $\mu>0, f$ is a forcing function, and $|\cdot|$ denotes the Euclidean norm on $\mathbf{R}^{n}$. A brief physical background of (1) is given in our previous paper [1].

For damped linear or semilinear systems, there are many books and articles about the well-posedness with applications to various dynamic system's topics (cf. [2-4], etc.) with semigroup or unified variational treatments. However, the quasilinear cases like (1) require more manipulations in the analysis of systems, because the systems like (1) are very much model-dependent due to the strong nonlinearity.

Equation (1) is proposed in Kobayashi et al. [5] and the well-posedness of strongly regular solutions is studied by using the resolvent estimates of linearized operators in a modified Banach space. Besides, the well-posedness of less regular solutions is proved in [1], called weak solutions in the framework of the variational method in Dautray and Lions [3]. Based on these results, we have treated the associated optimal control and identification problems in [6] and [7], respectively. Furthermore, in [8] we have extended the results in [1] to more general quasilinear nonautonomous wave equation with strong damping term.

In this paper, our concerned model is given by the following problem:

$$
\begin{aligned}
\frac{\partial^{2} y}{\partial t^{2}}-\nabla \cdot\left(\frac{\nabla y}{\sqrt{1+|\nabla y|^{2}}}\right)-\mu \Delta \frac{\partial y}{\partial t} & =\mathbf{U} y+f \quad \text { in } Q, \\
y & =0 \quad \text { on } \Sigma, \\
y(0, x) & =y_{0}(x), \\
\frac{\partial y}{\partial t}(0, x) & =y_{1}(x)
\end{aligned}
$$

in $\Omega$, 
where $\mathbf{U}$ is a bilinear forcing term which is usually referred to as bilinear control variable acting as a multiplier of the displacement term.

Bilinear optimal control problems with the state equation being a linear first or second PDEs such as reaction diffusion equation or Kirchhoff plate equation are studied by some authors (see [9-12] and references therein).

For future work, we will study bilinear optimal control problem with state equation (2). Then we must be faced with many difficulties because of the quasilinear term in (2). However, more regular solution of (2) corresponding to more regular data than weak one enables us to overcome these difficulties. This is motivation of this paper.

As is recognized, existence of regular solution of a quasilinear PDE is also quite model-dependent due to the strong nonlinearity.

We briefly summarize this paper as follows. At first, referring to the results in [1], we shall prove the wellposedness of weak solution of (2). Secondly, we shall analyze (2) in higher regularity than in [1] by employing newly constructed energy equality for (2). Finally, we shall prove by exploiting the well-posedness of weak solution of (2) that the regular solution of (2) is continuous with respect to regular data.

The most difficult part of the existence proof is to show the strong convergence of nonlinear terms, and the part is completed by using the argument in [3, p. 569]. This is another novelty of the paper.

\section{Notations and Main Results}

If $X$ is a Banach space, we denote by $X^{\prime}$ its topological dual and by $\langle\cdot, \cdot\rangle_{X^{\prime}, X}$ the duality pairing between $X^{\prime}$ and $X$. We introduce the following abbreviations:

$$
\begin{aligned}
L^{p} & =L^{p}(\Omega), \\
H^{k} & =H^{k}(\Omega), \\
\|\cdot\|_{p} & =\|\cdot\|_{L^{p}},
\end{aligned}
$$

with $p \geq 1$. And $H_{0}^{k}$ mean the completions of $C_{0}^{\infty}(\Omega)$ in $H^{k}$ for $k \geq 1$. Let $D(\Delta)=H^{2} \cap H_{0}^{1}$. If we denote the scalar product on $L^{2}$ by $(\cdot, \cdot)_{2}$, then the scalar products on $H_{0}^{1}$ and $D(\Delta)$ are given as follows:

$$
\begin{gathered}
((\psi, \phi))_{H_{0}^{1}}=(\nabla \psi, \nabla \phi)_{2}, \quad \forall \psi, \phi \in H_{0}^{1} ; \\
((\psi, \phi))_{D(\Delta)}=(\Delta \psi, \Delta \phi)_{2}, \quad \forall \psi, \phi \in D(\Delta) .
\end{gathered}
$$

Then obviously,

$$
\begin{gathered}
\|\psi\|_{H_{0}^{1}}=\|\nabla \psi\|_{2}, \quad \forall \psi \in H_{0}^{1}, \\
\|\phi\|_{D(\Delta)}=\|\Delta \phi\|_{2}, \quad \forall \phi \in D(\Delta) .
\end{gathered}
$$

The duality pairing between $H_{0}^{1}$ and $H^{-1}$ is denoted by $\langle\phi, \psi\rangle_{1,-1}$.
It is clear that

$$
D(\Delta) \hookrightarrow H_{0}^{1} \hookrightarrow L^{2} \hookrightarrow H^{-1} \hookrightarrow D(\Delta)^{\prime},
$$

and each space is dense in the following one and the injections are continuous.

Related to the nonlinear term in (2), we define the function $G: \mathbf{R}^{n} \rightarrow \mathbf{R}^{n}$ by $G(x)=x / \sqrt{1+|x|^{2}}, x \in \mathbf{R}^{n}$. Then it is easily verified that

$$
|G(x)-G(y)| \leq 2|x-y|, \quad \forall x, y \in \mathbf{R}^{n} .
$$

The nonlinear operator $G(\nabla \cdot): H_{0}^{1} \rightarrow\left[L^{\infty}\right]^{n}$ is defined by

$$
G(\nabla \phi)(x)=\frac{\nabla \phi(x)}{\sqrt{1+|\nabla \phi(x)|^{2}}}, \quad \text { a.e. } x \in \Omega, \forall \phi \in H_{0}^{1} \text {. }
$$

By the definition of $G(\nabla \cdot)$ in (8), we have the following useful property on $G(\nabla \cdot)$ :

$$
\begin{array}{cl}
\|G(\nabla \phi)\|_{2} \leq\|\nabla \phi\|_{2}, & \\
\|G(\nabla \phi)-G(\nabla \psi)\|_{2} \leq 2\|\nabla \phi-\nabla \psi\|_{2}, & \\
\forall \phi, \psi \in H_{0}^{1} .
\end{array}
$$

The solution space $W(0, T)$ for weak solutions of $(2)$ is defined by

$$
\begin{aligned}
& W(0, T)=\left\{g \mid g \in L^{2}\left(0, T ; H_{0}^{1}\right), g^{\prime}\right. \\
& \left.\quad \in L^{2}\left(0, T ; H_{0}^{1}\right), g^{\prime \prime} \in L^{2}\left(0, T ; H^{-1}\right)\right\}
\end{aligned}
$$

endowed with a norm

$$
\begin{aligned}
& \|g\|_{W(0, T)} \\
& =\left(\|g\|_{L^{2}\left(0, T ; H_{0}^{1}\right)}^{2}+\left\|g^{\prime}\right\|_{L^{2}\left(0, T ; H_{0}^{1}\right)}^{2}+\left\|g^{\prime \prime}\right\|_{L^{2}\left(0, T ; H^{-1}\right)}^{2}\right)^{1 / 2}
\end{aligned}
$$

where $g^{\prime}$ and $g^{\prime \prime}$ denote the first- and second-order distributive derivatives of $g$. We remark that $W(0, T)$ is continuously embedded in $C\left([0, T] ; H_{0}^{1}\right) \cap C^{1}\left([0, T] ; L^{2}\right)$ (cf. Dautray and Lions [3, p. 555]).

Definition 1. A function $y$ is said to be a weak solution of (2) if $y \in W(0, T)$ and $y$ satisfies

$$
\begin{array}{r}
\left\langle y^{\prime \prime}(\cdot), \phi\right\rangle_{-1,1}+(G(\nabla y(\cdot)), \nabla \phi)_{2}+\mu\left(\nabla y^{\prime}(\cdot), \nabla \phi\right)_{2} \\
=(\mathbf{U}(\cdot) y(\cdot), \phi)_{2}+\langle f(\cdot), \phi\rangle_{-1,1} \\
\forall \phi \in H_{0}^{1} \text { in the sense of } \mathscr{D}^{\prime}(0, T),
\end{array}
$$

$$
\begin{aligned}
& y(0)=y_{0} \in H_{0}^{1}, \\
& y^{\prime}(0)=y_{1} \in L^{2} .
\end{aligned}
$$

The following theorem gives the fundamental results on existence, uniqueness, and regularity of weak solutions of (2). 
Theorem 2. Assume that $\left(y_{0}, y_{1}, f\right) \in H_{0}^{1} \times L^{2} \times L^{2}\left(0, T ; H^{-1}\right)$, and $\mathbf{U} \in L^{\infty}(Q)$. Then problem (2) has a unique weak solution $y \in W(0, T)$. Moreover, the solution mapping $p=$ $\left(y_{0}, y_{1}, f, \mathbf{U}\right) \rightarrow y(p)$ of $\mathscr{P} \equiv H_{0}^{1} \times L^{2} \times L^{2}\left(0, T ; H^{-1}\right) \times L^{\infty}(Q)$ into $W(0, T)$ is locally Lipschitz continuous.

Indeed, let $p_{1}=\left(y_{0}^{1}, y_{1}^{1}, f_{1}, \mathbf{U}_{1}\right) \in \mathscr{P}$ and $p_{2}=\left(y_{0}^{2}\right.$, $\left.y_{1}^{2}, f_{2}, \mathbf{U}_{2}\right) \in \mathscr{P}$. We prove Theorem 2 by showing the inequality

$$
\begin{gathered}
\left\|y\left(p_{1}\right)-y\left(p_{2}\right)\right\|_{W(0, T)} \leq C\left(\left\|\nabla\left(y_{0}^{1}-y_{0}^{2}\right)\right\|_{2}^{2}\right. \\
+\left\|y_{1}^{1}-y_{1}^{2}\right\|_{2}^{2}+\left\|f_{1}-f_{2}\right\|_{L^{2}\left(0, T ; H^{-1}\right)}^{2} \\
\left.+\left\|\mathbf{U}_{\mathbf{1}}-\mathbf{U}_{\mathbf{2}}\right\|_{L^{\infty}(Q)}^{2}\right)^{1 / 2} \equiv C\left\|p_{1}-p_{2}\right\|_{\mathscr{P}},
\end{gathered}
$$

where $C>0$ is a constant depending on data.

Next we introduce the solution space $S(0, T)$ for strong solutions of (2) defined by

$$
\begin{gathered}
S(0, T)=\left\{g \mid g \in L^{2}(0, T ; D(\Delta)), g^{\prime}\right. \\
\left.\in L^{2}(0, T ; D(\Delta)), g^{\prime \prime} \in L^{2}(Q)\right\}
\end{gathered}
$$

endowed with a norm

$$
\begin{aligned}
& \|g\|_{S(0, T)} \\
& \quad=\left(\|g\|_{L^{2}(0, T ; D(\Delta))}^{2}+\left\|g^{\prime}\right\|_{L^{2}(0, T ; D(\Delta))}^{2}+\left\|g^{\prime \prime}\right\|_{L^{2}(\mathrm{Q})}^{2}\right)^{1 / 2},
\end{aligned}
$$

where $g^{\prime}$ and $g^{\prime \prime}$ denote the first- and second-order distributive derivatives of $g$. We remark also from Dautray and Lions [3, p. 555] that $S(0, T)$ is continuously embedded in $C([0, T] ; D(\Delta)) \cap C^{1}\left([0, T] ; H_{0}^{1}\right)$.

Definition 3. A function $y$ is said to be a strong solution of (2) if $y \in S(0, T)$ and $y$ satisfies

$$
\begin{aligned}
& y^{\prime \prime}(t)-\nabla \cdot G(\nabla y(t))-\mu \Delta y^{\prime}(t) \\
& \quad=\mathbf{U}(t) y(t)+f(t), \quad \text { a.e. } t \in[0, T], \\
& y(0)=y_{0} \in D(\Delta), \\
& y^{\prime}(0)=y_{1} \in H_{0}^{1} .
\end{aligned}
$$

The next theorem gives a well-posedness result for strong solutions of (2).

Theorem 4. Assume that $\left(y_{0}, y_{1}, f\right) \in D(\Delta) \times H_{0}^{1} \times L^{2}(Q)$, and $\mathbf{U} \in L^{\infty}(Q)$. Then (2) has a unique strong solution $y \in S(0, T)$ and it satisfies

$$
\begin{aligned}
& \|y\|_{S(0, T)} \\
& \quad \leq C\left(\left\|y_{0}\right\|_{D(\Delta)}^{2}+\left\|y_{1}\right\|_{H_{0}^{1}}^{2}+\|f\|_{L^{2}(Q)}^{2}+\|\mathbf{U}\|_{L^{\infty}(Q)}^{2}\right)^{1 / 2},
\end{aligned}
$$

where $C$ is a constant depending on data.
Now we give the result on the continuous dependence of strong solutions of (2) on $p=\left(y_{0}, y_{1}, f, \mathbf{U}\right)$. Let $\mathscr{F}$ be a product space defined by

$$
\mathscr{F}=D(\Delta) \times H_{0}^{1} \times L^{2}(Q) \times L^{\infty}(Q)
$$

endowed with a norm

$$
\begin{aligned}
& \|p\|_{\mathscr{F}} \\
& \quad=\left(\left\|y_{0}\right\|_{D(\Delta)}^{2}+\left\|y_{1}\right\|_{H_{0}^{1}}^{2}+\|f\|_{L^{2}(\mathrm{Q})}^{2}+\|\mathbf{U}\|_{L^{\infty}(\mathrm{Q})}^{2}\right)^{1 / 2} .
\end{aligned}
$$

For each $p=\left(y_{0}, y_{1}, f, \mathbf{U}\right) \in \mathscr{F}$ we have a strong solution $y=y(p) \in S(0, T)$ of (2) by Theorem 4. Thus, we can define the solution mapping $p=\left(y_{0}, y_{1}, f, \mathbf{U}\right) \rightarrow y(p)$ of $\mathscr{F}$ into $S(0, T)$.

Theorem 5. The nonlinear solution mapping $p=\left(y_{0}, y_{1}\right.$, $f, \mathbf{U}) \rightarrow y(p)$ of $\mathscr{F}$ into $S(0, T)$ of $(2)$ is continuous.

Throughout this paper, we will use $C$ as a generic constant and omit writing the integral variables in any definite integrals without confusion.

\section{Proof of Main Results}

Proof of Theorem 2. Since $\mathbf{U}_{i} \in L^{\infty}(Q)(i=1,2)$, by the results in [1], we can deduce that the weak solutions $y\left(p_{i}\right)$ of (2) corresponding to $p_{i}(i=1,2)$ exist in $W(0, T)$ such that

$$
\begin{aligned}
& \left\|y\left(p_{i}\right)\right\|_{W(0, T)} \\
& \quad \leq C\left(\left\|\nabla y_{0}^{i}\right\|_{2}^{2}+\left\|y_{1}^{i}\right\|_{2}^{2}+\left\|f_{i}\right\|_{L^{2}\left(0, T ; H^{-1}\right)}^{2}\right)^{1 / 2} \\
& \quad \leq C\left\|p_{i}\right\|_{\mathscr{P}} \quad(i=1,2) .
\end{aligned}
$$

We denote $y_{1}-y_{2} \equiv y\left(p_{1}\right)-y\left(p_{2}\right)$ by $\psi$. Then, we can get from (2) that $\psi$ satisfies the following equation in weak sense:

$$
\begin{aligned}
\psi^{\prime \prime}-\mu \Delta \psi^{\prime}= & \nabla \cdot G\left(\nabla y_{1}\right)-\nabla \cdot G\left(\nabla y_{2}\right)+\mathbf{U}_{1} \psi+\mathbf{U} y_{2} \\
& +\mathbf{f} \quad \text { in } Q, \\
\psi & =0 \text { on } \Sigma, \\
\psi(0)= & y_{0}^{1}-y_{0}^{2}, \\
\psi^{\prime}(0)= & y_{1}^{1}-y_{1}^{2}
\end{aligned}
$$

in $\Omega$,

where $\mathbf{U}=\mathbf{U}_{1}-\mathbf{U}_{2}$ and $\mathbf{f}=f_{1}-f_{2}$.

We multiply (21) by $\psi^{\prime}+\psi$ to have

$$
\begin{aligned}
& \frac{1}{2} \frac{d}{d t}\left(\left\|\psi^{\prime}(t)\right\|_{2}^{2}+\mu\|\nabla \psi(t)\|_{2}^{2}\right)+\mu\left\|\nabla \psi^{\prime}(t)\right\|_{2}^{2} \\
& \quad=-\left(G\left(\nabla y_{1}(t)\right)-G\left(\nabla y_{2}(t)\right), \nabla\left(\psi^{\prime}(t)+\psi(t)\right)\right)_{2}
\end{aligned}
$$




$$
\begin{aligned}
& +\left(\mathbf{U}_{1}(t) \psi(t)+\mathbf{U}(t) y_{2}(t), \psi^{\prime}(t)+\psi(t)\right)_{2} \\
& -\left\langle\psi^{\prime \prime}(t), \psi(t)\right\rangle_{-1,1} \\
& +\left\langle\mathbf{f}(t), \psi^{\prime}(t)-\psi(t)\right\rangle_{-1,1} .
\end{aligned}
$$

By integrating (22) over $[0, t]$, we obtain

$$
\begin{aligned}
\left\|\psi^{\prime}(t)\right\|_{2}^{2}+2 \mu \int_{0}^{t}\left\|\nabla \psi^{\prime}\right\|_{2}^{2} d s+\mu\|\nabla \psi(t)\|_{2}^{2} \\
=\left\|\psi^{\prime}(0)\right\|_{2}^{2}+\mu\|\nabla \psi(0)\|_{2}^{2} \\
\quad-2 \int_{0}^{t}\left(G\left(\nabla y_{1}\right)-G\left(\nabla y_{2}\right), \nabla\left(\psi^{\prime}+\psi\right)\right)_{2} d s \\
+2 \int_{0}^{t}\left(\mathbf{U}_{1} \psi+\mathbf{U} y_{2}, \psi^{\prime}+\psi\right)_{2} d s \\
\quad-2\left(\psi^{\prime}(t), \psi(t)\right)_{2}+2\left(\psi^{\prime}(0), \psi(0)\right)_{2} \\
+2 \int_{0}^{t}\left\|\psi^{\prime}\right\|_{2}^{2} d s+2 \int_{0}^{t}\left\langle\mathbf{f}, \psi^{\prime}+\psi\right\rangle_{-1,1} d s .
\end{aligned}
$$

Let $\epsilon>0$ be an arbitrary real number. Then, by (9), (20), and the Schwartz inequality we can obtain the following:

$$
\begin{aligned}
& \left|2 \int_{0}^{t}\left(G\left(\nabla y_{1}\right)-G\left(\nabla y_{2}\right), \nabla\left(\psi^{\prime}+\psi\right)\right)_{2} d s\right| \\
& \leq 4 \int_{0}^{t}\|\nabla \psi\|_{2}\left\|\nabla \psi^{\prime}\right\|_{2} d s+4 \int_{0}^{t}\|\nabla \psi\|_{2}^{2} d s \\
& \leq\left(\frac{4}{\epsilon}+4\right) \int_{0}^{t}\|\nabla \psi\|_{2}^{2} d s+\epsilon \int_{0}^{t}\left\|\nabla \psi^{\prime}\right\|_{2}^{2} d s \\
& \left|2 \int_{0}^{t}\left(\mathbf{U}_{1} \psi+\mathbf{U} y_{2}, \psi^{\prime}+\psi\right)_{2} d s\right| \\
& \leq 2 \int_{0}^{t}\left(\left\|\mathbf{U}_{1} \psi\right\|_{2}+\left\|\mathbf{U} y_{2}\right\|_{2}\right)\left(\left\|\psi^{\prime}\right\|_{2}+\|\psi\|_{2}\right) d s \\
& \leq 2\left\|\mathbf{U}_{1}\right\|_{L^{\infty}(Q)} \int_{0}^{t}\left(\|\psi\|_{2}\left\|\psi^{\prime}\right\|_{2}+\|\psi\|_{2}^{2}\right) d s \\
& +\int_{0}^{t}\left\|\mathbf{U} y_{2}\right\|_{2}^{2} d s+2 \int_{0}^{t}\left(\|\psi\|_{2}^{2}+\left\|\psi^{\prime}\right\|_{2}^{2}\right) d s \\
& \leq C \int_{0}^{t}\left(\|\nabla \psi\|_{2}^{2}+\left\|\psi^{\prime}\right\|_{2}^{2}\right) d s+\|\mathbf{U}\|_{L^{\infty}(\mathrm{Q})}^{2}\left\|y_{2}\right\|_{L^{2}(\mathrm{Q})}^{2} \\
& \leq C\left(\int_{0}^{t}\left(\|\nabla \psi\|_{2}^{2}+\left\|\psi^{\prime}\right\|_{2}^{2}\right) d s+\|\mathbf{U}\|_{L^{\infty}(\mathrm{Q})}^{2}\left\|y_{2}\right\|_{W(0, T)}^{2}\right) \\
& \leq C\left(\int_{0}^{t}\left(\|\nabla \psi\|_{2}^{2}+\left\|\psi^{\prime}\right\|_{2}^{2}\right) d s+\|\mathbf{U}\|_{L^{\infty}(\mathrm{Q})}^{2}\left\|p_{2}\right\|_{\mathscr{P}}^{2}\right) \\
& \left|2 \int_{0}^{t}\left\langle f, \psi^{\prime}+\psi\right\rangle_{-1,1} d s\right| \\
& \leq 2 \int_{0}^{t}\|f\|_{H^{-1}}\left(\left\|\nabla \psi^{\prime}\right\|_{2}+\|\nabla \psi\|_{2}\right) d s
\end{aligned}
$$

$$
\begin{aligned}
& \leq\left(\frac{1}{\epsilon}+1\right)\|f\|_{L^{2}\left(0, T ; H^{-1}\right)}^{2}+\epsilon \int_{0}^{t}\left\|\nabla \psi^{\prime}\right\|_{2}^{2} d s \\
&+\int_{0}^{t}\|\nabla \psi\|_{2}^{2} d s ; \\
& 2\left|\left(\psi^{\prime}(t), \psi(t)\right)_{2}\right| \leq 2\left\|\psi^{\prime}(t)\right\|_{2}\left\|\psi(0)+\int_{0}^{t} \psi^{\prime} d s\right\|_{2} \\
& \leq \epsilon\left\|\psi^{\prime}(t)\right\|_{2}^{2}+\frac{1}{\epsilon}\left\|\psi(0)+\int_{0}^{t} \psi^{\prime} d s\right\|_{2}^{2} \\
& \leq \epsilon\left\|\psi^{\prime}(t)\right\|_{2}^{2}+\frac{2}{\epsilon}\|\psi(0)\|_{2}^{2}+\frac{2 T}{\epsilon} \int_{0}^{t}\left\|\psi^{\prime}\right\|_{2}^{2} d s .
\end{aligned}
$$

We also note that $\left|2\left(\psi^{\prime}(0), \psi(0)\right)_{2}\right| \leq C\left(\|\nabla \psi(0)\|_{2}^{2}+\left\|\psi^{\prime}(0)\right\|_{2}^{2}\right)$. Therefore, from (23) and (24), we can obtain the following inequality:

$$
\begin{gathered}
(1-\epsilon)\left\|\psi^{\prime}(t)\right\|_{2}^{2}+\mu\|\nabla \psi(t)\|_{2}^{2}+(2 \mu-2 \epsilon) \\
\cdot \int_{0}^{t}\left\|\nabla \psi^{\prime}\right\|_{2}^{2} d s \leq C\left(1+\frac{1+T}{\epsilon}+\left\|p_{2}\right\|_{\mathscr{P}}^{2}\right) \\
\cdot\left(\|\nabla \psi(0)\|_{2}^{2}+\left\|\psi^{\prime}(0)\right\|_{2}^{2}+\|\mathbf{f}\|_{L^{2}\left(0, T ; H^{-1}\right)}^{2}\right. \\
\left.+\|\mathbf{U}\|_{L^{\infty}(Q)}^{2}+\int_{0}^{t}\left(\left\|\psi^{\prime}\right\|_{2}^{2}+\|\nabla \psi\|_{2}^{2}\right) d s\right) .
\end{gathered}
$$

If we choose $\epsilon=\min \{1 / 2, \mu / 2\}$, then by Bellman-Gronwall's inequality it follows that

$$
\begin{gathered}
\left\|\psi^{\prime}(t)\right\|_{2}^{2}+\|\nabla \psi(t)\|_{2}^{2}+\int_{0}^{t}\left\|\nabla \psi^{\prime}\right\|_{2}^{2} d s \leq C\left(T, p_{2}\right) \\
\cdot\left(\|\nabla \psi(0)\|_{2}^{2}+\left\|\psi^{\prime}(0)\right\|_{2}^{2}+\|\mathbf{f}\|_{L^{2}\left(0, T ; H^{-1}\right)}^{2}\right. \\
\left.+\|\mathbf{U}\|_{L^{\infty}(Q)}^{2}\right)=C\left(T, p_{2}\right)\left\|p_{1}-p_{2}\right\|_{\mathscr{P}}^{2}
\end{gathered}
$$

By (21) and (26) we have

$$
\begin{aligned}
& \left\|\psi^{\prime \prime}\right\|_{L^{2}\left(0, T ; H^{-1}\right)} \leq C\left(\left\|\nabla \psi^{\prime}\right\|_{L^{2}(\mathrm{Q})}\right. \\
& \quad+\left\|G\left(\nabla y_{1}\right)-G\left(\nabla y_{2}\right)\right\|_{L^{2}(\mathrm{Q})}+\|\psi\|_{L^{2}(\mathrm{Q})} \\
& \left.\quad+\|\mathbf{U}\|_{L^{\infty}(\mathrm{Q})}\left\|y_{2}\right\|_{L^{2}(\mathrm{Q})}+\|\mathbf{f}\|_{L^{2}\left(0, T ; H^{-1}\right)}\right) \\
& \quad \leq C\left(\left\|\nabla \psi^{\prime}\right\|_{L^{2}(\mathrm{Q})}+\|\nabla \psi\|_{L^{2}(\mathrm{Q})}\right. \\
& \left.\quad+\|\mathbf{U}\|_{L^{\infty}(\mathrm{Q})}\left\|y_{2}\right\|_{W(0, T)}+\|\mathbf{f}\|_{L^{2}\left(0, T ; H^{-1}\right)}\right) \\
& \quad \leq C_{1}\left(T, p_{2}\right)\left(\left\|p_{1}-p_{2}\right\|_{\mathscr{P}}+\|\mathbf{U}\|_{L^{\infty}(Q)}\left\|p_{2}\right\|_{\mathscr{P}}\right. \\
& \left.\quad+\|\mathbf{f}\|_{L^{2}\left(0, T ; H^{-1}\right)}\right) \leq C_{2}\left(T, p_{2}\right)\left\|p_{1}-p_{2}\right\|_{\mathscr{P}} .
\end{aligned}
$$

Finally, by combining (26) and (27) we obtain (13).

This completes the proof. 
Lemma 6. Let $X, Y$, and $Z$ be Banach spaces such that the imbeddings $X \subset Y \subset Z$ are continuous and the imbedding $X \subset Y$ is compact. Then a bounded set of $W^{1, \infty}(0, T ; X, Z)=$ $\left\{g \mid g \in L^{\infty}(0, T ; X), g^{\prime} \in L^{\infty}(0, T ; Z)\right\}$ is relatively compact in $C([0, T] ; Y)$.

Proof. See Simon [13].

Proof of Theorem 4. We divide the proof into three steps.

Step 1 (approximate solutions and a priori estimates). We construct approximate solutions of (2) by a Faedo-Galerkin's procedure. Since $D(\Delta)$ is separable, there exists a complete orthonormal system $\left\{w_{m}\right\}_{m=1}^{\infty}$ in $L^{2}$ such that $\left\{w_{m}\right\}_{m=1}^{\infty}$ is free and total in $D(\Delta)$. For each $m \in \mathbf{N}$ we can define an approximate solution of (2) by

$$
y_{m}(t)=\sum_{j=1}^{m} g_{j m}(t) w_{j}
$$

where $y_{m}(t)$ satisfies (2). Then (2) can be written as $m$ vector differential equations

$$
\widetilde{E} \frac{d^{2}}{d t^{2}} \vec{g}_{m}+\widetilde{G}\left(\nabla y_{m}\right) \vec{g}_{m}+\mu \widetilde{\Delta} \frac{d}{d t} \vec{g}_{m}=\widetilde{\mathbf{U}} \vec{g}_{m}+\vec{f}_{m}
$$

with initial values

$$
\begin{aligned}
\vec{g}_{m}(0) & =\left[\left(y_{0}, w_{1}\right)_{2},\left(y_{0}, w_{2}\right)_{2}, \ldots,\left(y_{0}, w_{m}\right)_{2}\right]^{t}, \\
\frac{d}{d t} \vec{g}_{m}(0) & =\left[\left(y_{1}, w_{1}\right)_{2},\left(y_{1}, w_{2}\right)_{2}, \ldots,\left(y_{1}, w_{m}\right)_{2}\right]^{t} .
\end{aligned}
$$

Notations of (29) can be explained as follows:

$$
\begin{aligned}
& \vec{g}_{m}=\left[g_{1 m}, \ldots, g_{m m}\right]^{t}, \\
& \widetilde{E}=\left(\left(w_{i}, w_{j}\right)_{2}=\delta_{i j}: i=1, \ldots, m, j=1, \ldots, m\right) \\
& \equiv I_{m} \text {, } \\
& \widetilde{G}\left(\nabla y_{m}\right)=\left(\frac{1}{\sqrt{1+\left|\nabla y_{m}\right|^{2}}}\left(\nabla w_{i}, \nabla w_{j}\right)_{2}: i\right. \\
& =1, \ldots, m, j=1, \ldots, m) \text {, } \\
& \mu \widetilde{\Delta}=\left(\mu\left(\nabla w_{i}, \nabla w_{j}\right)_{2}: i=1, \ldots, m, j=1, \ldots, m\right), \\
& \widetilde{\mathbf{U}}=\left(\left(\mathbf{U} w_{i}, w_{j}\right)_{2}: i=1, \ldots, m, j=1, \ldots, m\right), \\
& \vec{f}_{m}=\left[\left(f, w_{1}\right)_{2}, \ldots,\left(f, w_{m}\right)_{2}\right]^{t},
\end{aligned}
$$

where $[\cdots]^{t}$ denotes the transpose of $[\cdots]$. Since $G(\nabla \cdot)$ : $H_{0}^{1} \rightarrow\left[L^{\infty}\right]^{n}$ is Lipschitz continuous and $\mathbf{U} \in L^{\infty}(Q)$, we can deduce by Carathéodory type existence theorem that the nonlinear vector differential equation (29) admits a unique solution $\left[g_{1 m}, g_{2 m}, \ldots, g_{m m}\right]^{t}$ on $[0, T]$. Hence, we can construct the approximate solution $y_{m}(t)$ of (2). Next we shall derive a priori estimates of $y_{m}(t)$.

By analogy with (22), we take $L^{2}$ product of the equations for approximate solutions $y_{m}(t)$ with $-\Delta y_{m}^{\prime}(t)-\Delta y_{m}(t)$ to have

$$
\begin{aligned}
\frac{1}{2} \frac{d}{d t} & \left(\left\|\nabla y_{m}^{\prime}(t)\right\|_{2}^{2}+\mu\left\|\Delta y_{m}(t)\right\|_{2}^{2}\right)+\mu\left\|\Delta y_{m}^{\prime}(t)\right\|_{2}^{2} \\
= & -\left(\nabla \cdot G\left(\nabla y_{m}(t)\right), \Delta y_{m}^{\prime}(t)+\Delta y_{m}(t)\right)_{2} \\
& -\left(\mathbf{U}(t) y_{m}(t), \Delta y_{m}^{\prime}(t)+\Delta y_{m}(t)\right)_{2} \\
& +\left(y_{m}^{\prime \prime}(t), \Delta y_{m}(t)\right)_{2} \\
& -\left(f(t), \Delta y_{m}^{\prime}(t)+\Delta y_{m}(t)\right)_{2} .
\end{aligned}
$$

By integrating (32) over $[0, t]$, we obtain

$$
\begin{aligned}
& \left\|\nabla y_{m}^{\prime}(t)\right\|_{2}^{2}+2 \mu \int_{0}^{t}\left\|\Delta y_{m}^{\prime}\right\|_{2}^{2} d s+\mu\left\|\Delta y_{m}(t)\right\|_{2}^{2} \\
& =\left\|\nabla y_{1 m}\right\|_{2}^{2}+\mu\left\|\Delta y_{0 m}\right\|_{2}^{2} \\
& \quad-2 \int_{0}^{t}\left(\nabla \cdot G\left(\nabla y_{m}\right), \Delta y_{m}^{\prime}+\Delta y_{m}\right)_{2} d s \\
& \quad-2 \int_{0}^{t}\left(\mathbf{U} y_{m}, \Delta y_{m}^{\prime}+\Delta y_{m}\right)_{2} d s \\
& \quad+2\left(y_{m}^{\prime}(t), \Delta y_{m}(t)\right)_{2}-2\left(y_{1 m}, \Delta y_{0 m}\right)_{2} \\
& +2 \int_{0}^{t}\left\|\nabla y_{m}^{\prime}\right\|_{2}^{2} d s-2 \int_{0}^{t}\left(f, \Delta y_{m}^{\prime}+\Delta y_{m}\right)_{2} d s .
\end{aligned}
$$

Here we note from the elliptic regularity theory that

$$
\begin{aligned}
\|\nabla \cdot G(\nabla \phi)\|_{2} & =\left\|\frac{\Delta \phi}{\sqrt{1+|\nabla \phi|^{2}}}-\sum_{i, j=1}^{n} \frac{\phi_{x_{i}} \phi_{x_{j}} \phi_{x_{i} x_{j}}}{\left(1+|\nabla \phi|^{2}\right)^{3 / 2}}\right\|_{2} \\
& \leq\|\Delta \phi\|_{2}+\sum_{i, j=1}^{n}\left\|\phi_{x_{i} x_{j}}\right\|_{2} \leq C\|\Delta \phi\|_{2}
\end{aligned}
$$

$\forall \phi \in D(\Delta)$

Thus, by (34) we can deduce for $\epsilon>0$

$$
\begin{aligned}
& \left|2 \int_{0}^{t}\left(\nabla \cdot G\left(\nabla y_{m}\right), \Delta y_{m}^{\prime}+\Delta y_{m}\right)_{2} d s\right| \\
& \quad \leq 2 \int_{0}^{t}\left\|\nabla \cdot G\left(\nabla y_{m}\right)\right\|_{2}\left\|\Delta y_{m}^{\prime}+\Delta y_{m}\right\|_{2} d s \\
& \quad \leq C \int_{0}^{t}\left\|\Delta y_{m}\right\|_{2}\left(\left\|\Delta y_{m}^{\prime}\right\|_{2}+\left\|\Delta y_{m}\right\|_{2}\right) d s \\
& \quad \leq \epsilon \int_{0}^{t}\left\|\Delta y_{m}^{\prime}\right\|_{2}^{2} d s+C \int_{0}^{t}\left\|\Delta y_{m}\right\|_{2}^{2} d s
\end{aligned}
$$




$$
\begin{aligned}
& 2\left|\left(y_{m}^{\prime}(t), \Delta y_{m}(t)\right)_{2}\right| \\
& \quad \leq 2\left\|\nabla y_{m}^{\prime}(t)\right\|_{2}\left\|\nabla\left(y_{0 m}+\int_{0}^{t} y_{m}^{\prime} d s\right)\right\|_{2} \\
& \quad \leq \epsilon\left\|\nabla y_{m}^{\prime}(t)\right\|_{2}^{2}+\frac{1}{\epsilon}\left\|\nabla\left(y_{0 m}+\int_{0}^{t} y_{m}^{\prime} d s\right)\right\|_{2}^{2} \\
& \quad \leq \epsilon\left\|\nabla y_{m}^{\prime}(t)\right\|_{2}^{2}+\frac{2}{\epsilon}\left\|\nabla y_{0 m}\right\|_{2}^{2}+\frac{2 T}{\epsilon} \int_{0}^{t}\left\|\nabla y_{m}^{\prime}\right\|_{2}^{2} d s .
\end{aligned}
$$

For other estimations of the terms in the RHS of (33) other than (35), we can follow the analogous process in the proof of Theorem 2 to get

$$
\begin{gathered}
\left\|\nabla y_{m}^{\prime}(t)\right\|_{2}^{2}+\int_{0}^{t}\left\|\Delta y_{m}^{\prime}\right\|_{2}^{2} d s+\left\|\Delta y_{m}(t)\right\|_{2}^{2} \\
\leq C\left(\left\|\nabla y_{1 m}\right\|_{2}^{2}+\left\|\Delta y_{0 m}\right\|_{2}^{2}+\|f\|_{L^{2}(Q)}^{2}\right. \\
\left.+\int_{0}^{t}\left(\left\|\nabla y_{m}^{\prime}\right\|_{2}^{2}+\left\|\Delta y_{m}\right\|_{2}^{2}\right) d s\right) .
\end{gathered}
$$

Therefore, it is shown by using Bellman-Gronwall's inequality that

$$
\begin{gathered}
\left\|\nabla y_{m}^{\prime}(t)\right\|_{2}^{2}+\left\|\Delta y_{m}(t)\right\|_{2}^{2}+\int_{0}^{t}\left\|\Delta y_{m}^{\prime}\right\|_{2}^{2} d s \\
\leq C\left(\left\|\Delta y_{0 m}\right\|_{2}^{2}+\left\|\nabla y_{1 m}\right\|_{2}^{2}+\|f\|_{L^{2}(\mathrm{Q})}^{2}\right),
\end{gathered}
$$

$\forall t \in[0, T]$.

And also from Theorem 2, (2), (34), and (37), we have

$$
\begin{aligned}
& \left\|y_{m}^{\prime \prime}\right\|_{L^{2}(\mathrm{Q})} \leq\left\|\nabla \cdot G\left(\nabla y_{m}\right)\right\|_{L^{2}(\mathrm{Q})}+\mu\left\|\Delta y_{m}^{\prime}\right\|_{L^{2}(\mathrm{Q})} \\
& +\left\|\mathbf{U} y_{m}\right\|_{L^{2}(\mathrm{Q})}+\|f\|_{L^{2}(\mathrm{Q})} \leq C\left(\left\|\Delta y_{m}\right\|_{L^{2}(\mathrm{Q})}\right. \\
& \left.+\left\|\Delta y_{m}^{\prime}\right\|_{L^{2}(\mathrm{Q})}+\|\mathbf{U}\|_{L^{\infty}(\mathrm{Q})}\left\|y_{m}\right\|_{L^{2}(\mathrm{Q})}+\|f\|_{L^{2}(\mathrm{Q})}\right) \\
& \quad \leq C\left(\left\|\Delta y_{m}\right\|_{L^{2}(\mathrm{Q})}+\left\|\Delta y_{m}^{\prime}\right\|_{L^{2}(\mathrm{Q})}\right. \\
& \left.+\|\mathbf{U}\|_{L^{\infty}(\mathrm{Q})}\left\|y_{m}\right\|_{W(0, T)}+\|f\|_{L^{2}(\mathrm{Q})}\right) \leq C(1 \\
& \left.+\left\|p_{m}\right\|_{\mathscr{P}}\right)\left(\left\|\Delta y_{0 m}\right\|_{2}+\left\|\nabla y_{1 m}\right\|_{2}+\|f\|_{L^{2}(\mathrm{Q})}\right. \\
& \left.+\|\mathbf{U}\|_{L^{\infty}(\mathrm{Q})}\right)
\end{aligned}
$$

where $p_{m}=\left(y_{0 m}, y_{1 m}, f, \mathbf{U}\right)$.

Step 2 (passage to the limits). Equations (37) and (38) imply that

$$
\left\{y_{m}\right\} \text { is bounded in } S(0, T)
$$

$$
\cap W^{1, \infty}\left(0, T ; D(\Delta), H_{0}^{1}\right) .
$$

And the nonlinear term $\nabla \cdot G\left(\nabla y_{m}\right)$ is bounded in $L^{\infty}(0$, $\left.T ; L^{2}\right)$. Hence, by the extraction theorem of Rellich, we can extract a subsequence $\left\{y_{m_{k}}\right\}$ of $\left\{y_{m}\right\}$ and find $y \in S(0, T) \cap$ $W^{1, \infty}\left(0, T ; D(\Delta), H_{0}^{1}\right)$ and $\mathscr{G} \in L^{\infty}\left(0, T ; L^{2}\right)$ such that

(i) $y_{m_{k}} \longrightarrow y$ weakly-star in $L^{\infty}(0, T ; D(\Delta))$ and weakly in $S(0, T)$,

(ii) $y_{m_{k}}^{\prime} \longrightarrow y^{\prime}$ weakly-star in $L^{\infty}\left(0, T ; H_{0}^{1}\right)$,

(iii) $\nabla \cdot G\left(\nabla y_{m_{k}}\right)$

$\longrightarrow \mathscr{G}$ weakly-star in $L^{\infty}\left(0, T ; L^{2}\right)$,

as $k \rightarrow \infty$. Since $D(\Delta) \hookrightarrow H_{0}^{1}$ is compact, we can apply Lemma 6 with $X=D(\Delta), Y=Z=H_{0}^{1}$ and AubinLions-Temam's compact imbedding theorem (cf. Temam [14, p. 274]) to (39) to verify that $\left\{y_{m}\right\}$ and $\left\{y_{m}^{\prime}\right\}$ are precompact in $C\left([0, T] ; H_{0}^{1}\right)$ and $L^{2}\left(0, T ; H_{0}^{1}\right)$, respectively. Hence, we can find a subsequence $\left\{y_{m_{k}}\right\} \subset\left\{y_{m}\right\}$, if necessary, such that

$$
\begin{aligned}
& y_{m_{k}} \longrightarrow y \quad \text { strongly in } C\left([0, T] ; H_{0}^{1}\right), \\
& y_{m_{k}}^{\prime} \longrightarrow y^{\prime} \text { strongly in } L^{2}\left(0, T ; H_{0}^{1}\right)
\end{aligned}
$$

as $k \rightarrow \infty$.

By the standard argument of Dautray and Lions [3, pp. 564-566], it can be verified that the limit $y$ of $\left\{y_{m_{k}}\right\}$ is a strong solution of the linear problem

$$
\begin{aligned}
y^{\prime \prime}-\mathscr{G}-\mu \Delta y^{\prime} & =\mathbf{U} y+f \quad \text { in } Q, \\
y & =0 \quad \text { on } \Sigma, \\
y(0, x) & =y_{0}, \\
y^{\prime}(0, x) & =y_{1},
\end{aligned}
$$

in $\Omega$.

Step 3 (strong convergence of approximate solutions). In order to prove that $y$ is a strong solution of (2), it is sufficient to prove $\mathscr{G}=\nabla \cdot G(\nabla y)$. For this, we shall show $y_{m}(t) \rightarrow$ $y(t)$ strongly in $D(\Delta)$ for all $t \in[0, T]$. To prove the strong convergence, we use the modified arguments in Dautray and Lions [3, pp. 579-581] and the classical compact imbedding theorem.

First as in (33), we take $L^{2}$ product equation (43) with $-\Delta y^{\prime}-\Delta y$ and integrate it over $[0, t]$ to have

$$
\begin{aligned}
& \left\|\nabla y^{\prime}(t)\right\|_{2}^{2}+2 \mu \int_{0}^{t}\left\|\Delta y^{\prime}\right\|_{2}^{2} d s+\mu\|\Delta y(t)\|_{2}^{2} \\
& =\left\|\nabla y_{1}\right\|_{2}^{2}+\mu\left\|\Delta y_{0}\right\|_{2}^{2}-2 \int_{0}^{t}\left(\mathscr{G}, \Delta y^{\prime}+\Delta y\right)_{2} d s \\
& \quad-2 \int_{0}^{t}\left(\mathbf{U} y, \Delta y^{\prime}+\Delta y\right)_{2} d s+2\left(y^{\prime}(t), \Delta y(t)\right)_{2}
\end{aligned}
$$




$$
\begin{aligned}
& -2\left(y_{1}, \Delta y_{0}\right)_{2}+2 \int_{0}^{t}\left\|\nabla y^{\prime}\right\|_{2}^{2} d s \\
& -2 \int_{0}^{t}\left(f, \Delta y^{\prime}+\Delta y\right)_{2} d s .
\end{aligned}
$$

By making use of the following trivial equalities:

$$
\begin{aligned}
\|\phi\|_{2}^{2}+\|\varphi\|_{2}^{2}= & \|\phi-\varphi\|_{2}^{2}+2(\phi, \varphi)_{2}, \quad \forall \phi, \varphi \in L^{2}, \\
\left(\phi_{1}, \varphi_{1}\right)_{2}= & \left(\phi_{1}-\phi_{2}, \varphi_{1}-\varphi_{2}\right)_{2}+\left(\phi_{1}, \varphi_{2}\right)_{2} \\
& +\left(\phi_{2}, \varphi_{1}-\varphi_{2}\right)_{2},
\end{aligned}
$$$$
\forall \phi_{i}, \varphi_{i} \in L^{2}(i=1,2),
$$

$$
\begin{aligned}
& +2 \sum_{i=1}^{3} \Phi_{m}^{i}(t)-2 \int_{0}^{t}\left(\nabla \cdot G\left(\nabla y_{m}\right)-\nabla\right. \\
& \left.\cdot G(\nabla y), \Delta \psi_{m}^{\prime}+\Delta \psi_{m}\right)_{2} d s
\end{aligned}
$$

where

$$
\begin{aligned}
& \Phi_{m}^{0}=\left\|\nabla y_{1 m}\right\|_{2}^{2}+\left\|\nabla y_{1}\right\|_{2}^{2}+\mu\left(\left\|\nabla y_{0 m}\right\|_{2}^{2}+\left\|\nabla y_{0}\right\|_{2}^{2}\right) \\
& -2\left(\left(y_{1 m}, \Delta y_{0 m}\right)_{2}+\left(y_{1}, \Delta y_{0}\right)_{2}\right) \text {, } \\
& \Phi_{m}^{1}(t)=-\left(\nabla y_{m}^{\prime}(t), \nabla y^{\prime}(t)\right)_{2}-\mu\left(\Delta y_{m}(t), \Delta y(t)\right)_{2} \\
& -2 \mu \int_{0}^{t}\left(\Delta y_{m}^{\prime}, \Delta y^{\prime}\right)_{2} d s, \\
& \Phi_{m}^{2}(t)=-\int_{0}^{t}\left(\mathbf{U} y_{m}, \Delta y_{m}^{\prime}+\Delta y_{m}\right)_{2} d s \\
& -\int_{0}^{t}\left(\mathbf{U} y, \Delta y^{\prime}+\Delta y\right)_{2} d s \\
& +\int_{0}^{t}\left\|\nabla y_{m}^{\prime}\right\|_{2}^{2} d s+\int_{0}^{t}\left\|\nabla y^{\prime}\right\|_{2}^{2} d s \\
& -\int_{0}^{t}\left(f, \Delta y_{m}^{\prime}+\Delta y_{m}\right)_{2} d s \\
& -\int_{0}^{t}\left(f, \Delta y^{\prime}+\Delta y\right)_{2} d s d s \\
& +\left(y_{m}^{\prime}(t), \Delta y_{m}(t)\right)_{2}+\left(y^{\prime}(t), \Delta y(t)\right)_{2}, \\
& \Phi_{m}^{3}(t)=-\int_{0}^{t}\left(\nabla \cdot G\left(\nabla y_{m}\right), \Delta y^{\prime}+\Delta y\right)_{2} d s \\
& -\int_{0}^{t}\left(\mathscr{G}, \Delta y^{\prime}+\Delta y\right)_{2} d s \\
& -\int_{0}^{t}\left(\nabla \cdot G(\nabla y), \Delta \psi_{m}^{\prime}+\Delta \psi_{m}\right)_{2} d s .
\end{aligned}
$$

Then by routine calculations in (52) together with (53), we can derive the following inequality:

$$
\begin{aligned}
& \left\|\nabla \psi_{m}^{\prime}(t)\right\|_{2}^{2}+\left\|\Delta \psi_{m}(t)\right\|_{2}^{2}+\int_{0}^{t}\left\|\Delta \psi_{m}^{\prime}\right\|_{2}^{2} d s \\
& \quad \leq C\left(\left|\Phi_{m}(t)\right|+\int_{0}^{t}\left\|\mathscr{R}_{m}\right\|_{2}^{2} d s\right)+C \int_{0}^{t}\left\|\Delta \psi_{m}\right\|_{2}^{2} d s .
\end{aligned}
$$

By applying the extended Bellman-Gronwall's inequality to (54), we deduce

$$
\begin{gathered}
\left\|\nabla \psi_{m}^{\prime}(t)\right\|_{2}^{2}+\left\|\Delta \psi_{m}(t)\right\|_{2}^{2}+\int_{0}^{t}\left\|\Delta \psi_{m}^{\prime}\right\|_{2}^{2} d s \\
\leq C\left(\left|\Phi_{m}(t)\right|+\left\|\mathscr{R}_{m}\right\|_{L^{2}(Q)}\right) \\
+C \int_{0}^{t}\left(\left|\Phi_{m}\right|+\left\|\mathscr{R}_{m}\right\|_{L^{2}(Q)}\right) d s .
\end{gathered}
$$


By virtue of the strong convergence of the initial values and (40)-(42), we can extract a subsequence $\left\{y_{m_{k}}\right\}$ of $\left\{y_{m}\right\}$ such that

$$
\begin{aligned}
\Phi_{m_{k}}^{0} \longrightarrow 2 & \left\|\nabla y_{1}\right\|_{2}^{2}+2 \mu\left\|\Delta y_{0}\right\|_{2}^{2}-4\left(y_{1}, \Delta y_{0}\right)_{2}, \\
\Phi_{m_{k}}^{1}(t) \longrightarrow & -\left\|\nabla y^{\prime}(t)\right\|_{2}^{2}-\mu\|\Delta y(t)\|_{2}^{2} \\
& -2 \mu \int_{0}^{t}\left\|\Delta y^{\prime}\right\|_{2}^{2} d s \\
\Phi_{m_{k}}^{2}(t) \longrightarrow & -2 \int_{0}^{t}\left(\mathbf{U} y, \Delta y^{\prime}+\Delta y\right)_{2} d s \\
& +2 \int_{0}^{t}\left\|\nabla y^{\prime}\right\|_{2}^{2} d s \\
& -2 \int_{0}^{t}\left(f, \Delta y^{\prime}+\Delta y\right)_{2} d s \\
& +2\left(y^{\prime}(t), \Delta y(t)\right)_{2}, \\
\Phi_{m_{k}}^{3}(t) \longrightarrow & -2 \int_{0}^{t}\left(\mathscr{G}, \Delta y^{\prime}+\Delta y\right)_{2} d s
\end{aligned}
$$

as $k \rightarrow \infty$. Therefore, in view of (44) we can deduce by the sum of limits in (56) that

$$
\Phi_{m_{k}}(t) \longrightarrow 0 \text { as } k \longrightarrow \infty .
$$

Also from (41), we can easily verify that there exists a subsequence $\left\{y_{m_{k}}\right\}$ of $\left\{y_{m}\right\}$ such that

$$
y_{m_{k} x_{i}} \longrightarrow y_{x_{i}} \text { a.e. } x \in \Omega, i=1, \ldots, n
$$

$$
\left(\psi_{m_{k}}, \psi_{m_{k}}^{\prime}\right) \longrightarrow(0,0) \quad \text { strongly in } C([0,
$$

as $k \rightarrow \infty$. From (49), (53), (62), and (63), it follows that

$$
\nabla \cdot G\left(\nabla y_{m_{k}}\right) \longrightarrow \nabla \cdot G(\nabla y) \quad \text { strongly in } L^{2}(Q)
$$

as $k \rightarrow \infty$. Thus, we readily have

$$
\mathscr{G}=\nabla \cdot G(\nabla y) .
$$

Therefore, we have proved the existence of a strong solution of (2). (17).

By similar estimations as in (37) and (38), we can show

The uniqueness of strong solutions is evident from the uniqueness of weak solutions.

Proof of Theorem 5. Let $y(p+\delta p)$ and $y(p)$ be the strong solutions of (2) corresponding to $p+\delta p=\left(y_{0}+\delta y_{0}, y_{1}+\right.$ $\left.\delta y_{1}, f+\delta f, \mathbf{U}+\delta \mathbf{U}\right) \in \mathscr{F}$ and $p=\left(y_{0}, y_{1}, f, \mathbf{U}\right) \in \mathscr{F}$, respectively. Set $\psi_{\delta}=y(p+\delta p)-y(p)$. Then $\psi_{\delta}$ satisfies

$$
\begin{aligned}
\psi_{\delta}^{\prime \prime}-\mu \Delta \psi_{\delta}^{\prime}= & \nabla \cdot G(\nabla y(p+\delta p))-\nabla \cdot G(\nabla y(p)) \\
& +\mathbf{U} \psi_{\delta}+\delta \mathbf{U} y(p+\delta p)+\delta f
\end{aligned}
$$

in $Q$, for all $t \in[0, T]$. Then it follows from (58) that

$$
\begin{array}{r}
\frac{1}{\sqrt{1+\left|\nabla y_{m_{k}}(t, x)\right|^{2}}} \longrightarrow \frac{1}{\sqrt{1+|\nabla y(t, x)|^{2}}} \\
\frac{y_{m_{k} x_{i}}(t, x) y_{m_{k} x_{j}}(t, x)}{\left(1+\left|\nabla y_{m_{k}}(t, x)\right|^{2}\right)^{3 / 2}} \longrightarrow \frac{y_{x_{i}}(t, x) y_{x_{j}}(t, x)}{\left(1+|\nabla y(t, x)|^{2}\right)^{3 / 2}} \\
\text { a.e. } x \in \Omega, \\
\text { a.e. } x \in \Omega
\end{array}
$$

for all $t \in[0, T]$. Hence, we have

$$
\lim _{k \rightarrow \infty} \mathscr{R}_{m_{k}}(t, x)=0 \quad \text { a.e. }(t, x) \in Q .
$$

Since

$$
\left|\mathscr{R}_{m}(t, x)\right| \leq 2|\Delta y(t, x)|+2 \sum_{i, j=1}^{n}\left|y_{x_{i} x_{j}}(t, x)\right|
$$

a.e. $(t, x) \in Q$,

we see from (60), (61), and the Lebesgue dominated convergence theorem that

$$
\mathscr{R}_{m_{k}} \longrightarrow 0 \text { strongly in } L^{2}(Q) \text { as } k \longrightarrow \infty \text {. }
$$

By applying (57) and (62) to (55) with $m=m_{k}$, we have

$D(\Delta)) \times\left(C\left([0, T] ; H_{0}^{1}\right) \cap L^{2}(0, T ; D(\Delta))\right)$

$$
\begin{aligned}
\psi_{\delta} & =0 \quad \text { on } \Sigma, \\
\psi_{\delta}(0, x) & =\delta y_{0}, \\
\psi_{\delta}^{\prime}(0, x) & =\delta y_{1},
\end{aligned}
$$

in $\Omega$

in the weak sense. Here we can repeat the same estimations in the proof of Theorem 4 mainly by performing $L^{2}(Q)$-product of (66) with $-\Delta \psi_{\delta}^{\prime}-\Delta \psi_{\delta}$ to obtain

$$
\begin{aligned}
& \left\|\nabla \psi_{\delta}^{\prime}(t)\right\|_{2}^{2}+\left\|\Delta \psi_{\delta}(t)\right\|_{2}^{2}+\int_{0}^{t}\left\|\Delta \psi_{\delta}^{\prime}\right\|_{2}^{2} d s \\
& \leq C\left(\int_{0}^{t}\left\|\mathscr{R}_{\delta}\right\|_{2}^{2} d s+\left\|\Delta\left(\delta y_{0}\right)\right\|_{2}^{2}+\left\|\nabla\left(\delta y_{1}\right)\right\|_{2}^{2}\right. \\
& \left.\quad+\|\delta \mathbf{U} y(p+\delta p)+\delta f\|_{L^{2}(\mathrm{Q})}^{2}\right) \leq C\left(\left\|\mathscr{R}_{\delta}\right\|_{L^{2}(\mathrm{Q})}^{2}\right. \\
& \quad+\left\|\Delta\left(\delta y_{0}\right)\right\|_{2}^{2}+\left\|\nabla\left(\delta y_{1}\right)\right\|_{2}^{2}
\end{aligned}
$$




$$
\begin{aligned}
& \left.+\|\delta \mathbf{U}\|_{L^{\infty}(\mathrm{Q})}^{2}\|y(p+\delta p)\|_{L^{2}(\mathrm{Q})}^{2}+\|\delta f\|_{L^{2}(\mathrm{Q})}^{2}\right) \\
& \leq C\left(\left\|\mathscr{R}_{\delta}\right\|_{L^{2}(\mathrm{Q})}^{2}+\left\|\Delta\left(\delta y_{0}\right)\right\|_{2}^{2}+\left\|\nabla\left(\delta y_{1}\right)\right\|_{2}^{2}\right. \\
& \left.+\|\delta \mathbf{U}\|_{L^{\infty}(\mathrm{Q})}^{2}\|p+\delta p\|_{\mathscr{P}}^{2}+\|\delta f\|_{L^{2}(\mathrm{Q})}^{2}\right) \leq C(1 \\
& \left.+\|p+\delta p\|_{\mathscr{P}}^{2}\right)\left(\left\|\mathscr{R}_{\delta}\right\|_{L^{2}(\mathrm{Q})}^{2}+\|\delta p\|_{\mathscr{F}}^{2}\right),
\end{aligned}
$$

where $\mathscr{R}_{\delta}$ is given by (51) in which $y_{m}$ and $y$ are replaced by $y(p+\delta p)$ and $y(p)$, respectively. By Theorem 2 , we know that

$$
\left\|\psi_{\delta}\right\|_{W(0, T)} \leq C\|\delta p\|_{\mathscr{P}} .
$$

Equation (68) immediately implies

$$
\begin{aligned}
\psi_{\delta} \longrightarrow & 0 \\
& \text { strongly in } C\left([0, T] ; H_{0}^{1}\right) \text { as } \delta p \longrightarrow 0 \text { in } \mathscr{P} .
\end{aligned}
$$

Whence from (58)-(62) we know that

$$
\mathscr{R}_{\delta} \longrightarrow 0 \text { strongly in } L^{2}(Q) \text { as } \delta p \longrightarrow 0 \text { in } \mathscr{P} \text {. }
$$

Finally by (66), (67), and (70), we can conclude that

$$
\psi_{\delta} \longrightarrow 0 \text { strongly in } S(0, T) \text { as } \delta p \longrightarrow 0 \text { in } \mathscr{F} \text {. }
$$

This completes the proof.

\section{Conflicts of Interest}

The author declares that there are no conflicts of interest regarding the publication of this article.

\section{Acknowledgments}

This research was supported by the Daegu University Research Grant 2014.

\section{References}

[1] J.-s. Hwang and S.-i. Nakagiri, "Weak solutions of the equation of motion of membrane with strong viscosity," Journal of the Korean Mathematical Society, vol. 44, no. 2, pp. 443-453, 2007.

[2] H. T. Banks, R. C. Smith, and Y. Wang, Smart Material Structures, Modeling, Estimation and Control, John Wiley and Sons, 1996.

[3] R. Dautray and J.-L. Lions, "Mathematical Analysis and Numerical Methods for Science and Technology," in Evolution Problems I, vol. 5, Springer-Verlag, 1992.

[4] R. Temam, Infinite-Dimensional Dynamical Systems in Mechanics and Physics, vol. 68 of Applied Mathematical Sciences, Springer, New York, NY, USA, 2nd edition, 1997.

[5] T. Kobayashi, H. Pecher, and Y. Shibata, "On a global in time existence theorem of smooth solutions to a nonlinear wave equation with viscosity," Mathematische Annalen, vol. 296, no. 2, pp. 215-234, 1993.

[6] J.-s. Hwang and S.-i. Nakagiri, "Optimal control problems for the equation of motion of membrane with strong viscosity," Journal of Mathematical Analysis and Applications, vol. 321, no. 1, pp. 327-342, 2006.
[7] J.-s. Hwang and S.-i. Nakagiri, "Parameter identification problem for the equation of motion of membrane with strong viscosity," Journal of Mathematical Analysis and Applications, vol. 342, no. 1, pp. 125-134, 2008.

[8] J.-s. Hwang, S.-i. Nakagiri, and H. Tanabe, "Solutions of quasilinear wave equation with strong and nonlinear viscosity," Journal of the Korean Mathematical Society, vol. 48, no. 4, pp. 867-885, 2011.

[9] A. Belmiloudi, "Bilinear minimax control problems for a class of parabolic systems with applications to control of nuclear reactors," Journal of Mathematical Analysis and Applications, vol. 327, no. 1, pp. 620-642, 2007.

[10] M. E. Bradley and S. Lenhart, "Bilinear spatial control of the velocity term in a Kirchhoff plate equation," Electronic Journal of Differential Equations, No. 27, 15 pages, 2001.

[11] M. E. Bradley, S. Lenhart, and J. Yong, "Bilinear optimal control of the velocity term in a Kirchhoff plate equation," Journal of Mathematical Analysis and Applications, vol. 238, no. 2, pp. 451467, 1999.

[12] S. Lenhart and M. Liang, "Bilinear optimal control for a wave equation with viscous damping," Houston Journal of Mathematics, vol. 26, no. 3, pp. 575-595, 2000.

[13] J. Simon, "Compact sets in the space Lp," Annali di Matematica Pura ed Applicata, vol. 146, no. 4, pp. 65-96, 1987.

[14] R. Temam, Navier-Stokes Equations, North-Holland, Amsterdam, The Netherlands, 1984. 


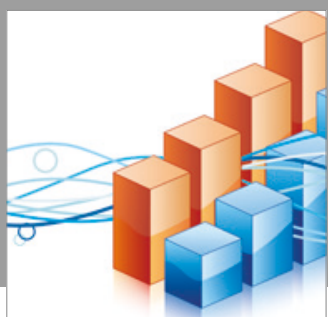

Advances in

Operations Research

vatersals

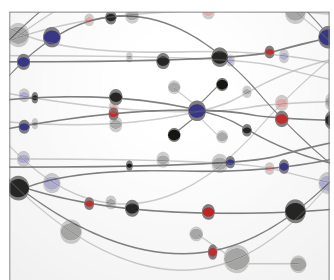

\section{The Scientific} World Journal
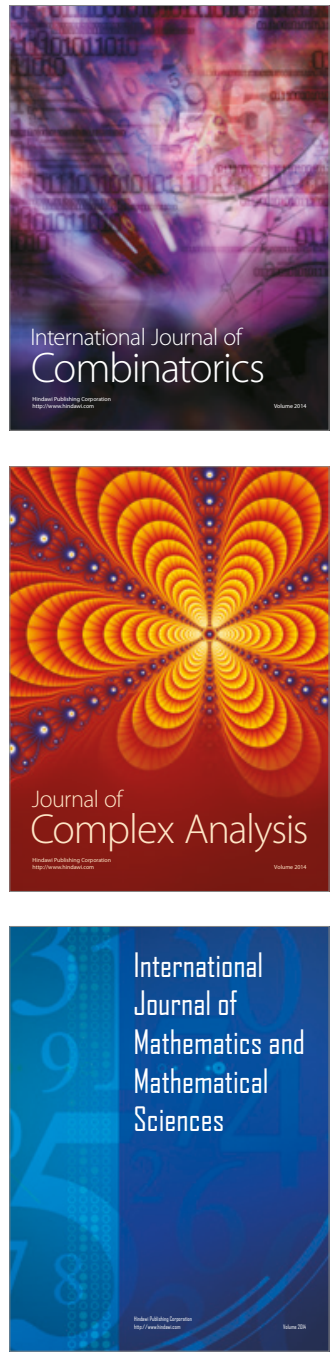
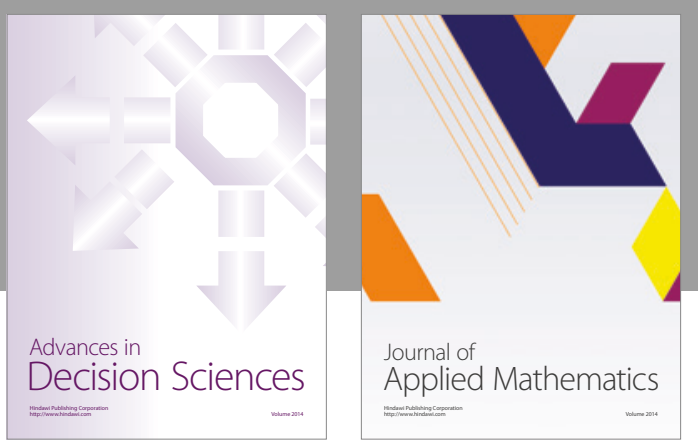

Algebra

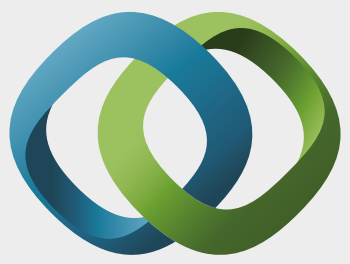

\section{Hindawi}

Submit your manuscripts at

https://www.hindawi.com
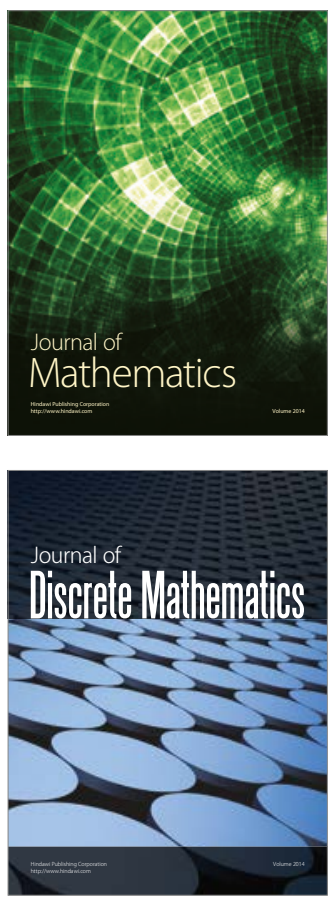

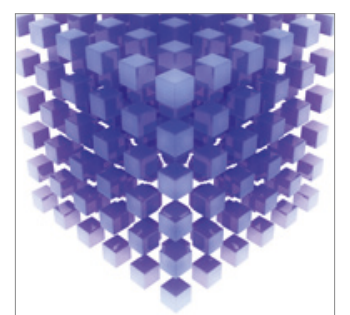

Mathematical Problems in Engineering
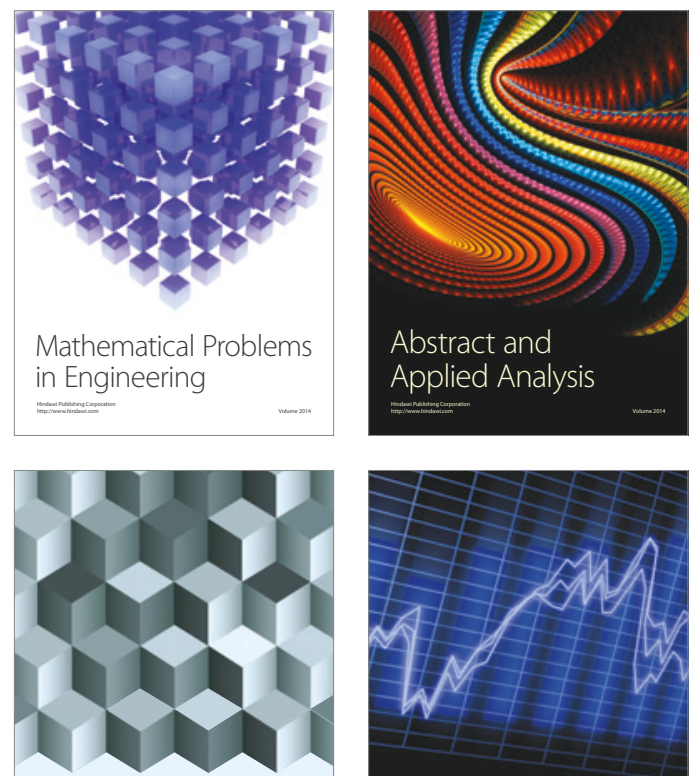

Journal of

Function Spaces

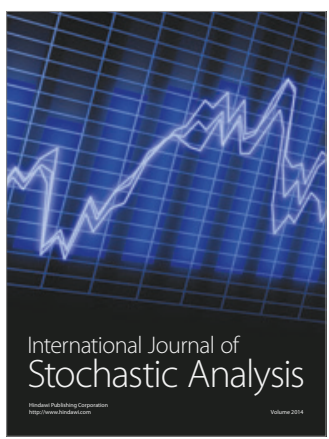

Probability and Statistics
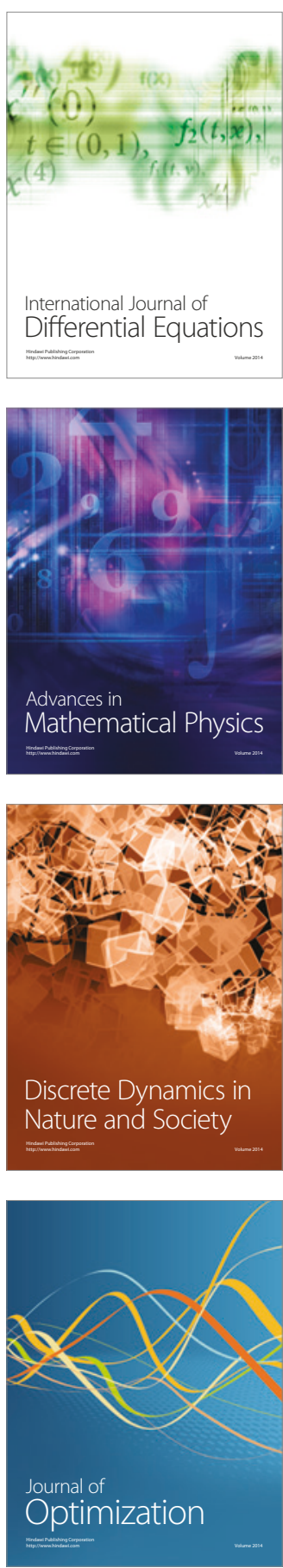